\title{
Prevalencia de Helicobacter pylori en un grupo de pacientes pediátricos infectados por el virus de la inmunodeficiencia humana
}

\author{
Julio Luarca, Fabio Parada*, Oscar Osorio, Julio Linares, Franco Pezzarossi, Ana Orantes, Guido Andretta, \\ Andrea Sandoval, Lashly Méndez, Fernando Gutiérrez, Luis Fernández, Wendoly Munguia
}

Facultad de Ciencias Médicas, Universidad San Carlos de Guatemala, Guatemala.

*Autor al que se dirige la correspondencia: enriquepc2@aol.com

Recibido: 12 de mayo 2015 / Revisión: 09 de junio 2015 / Aceptado: 03 de agosto 2015 / Disponible en línea: 24 de septiembre 2015

\section{Resumen}

Q e realizó un estudio cuantitativo, descriptivo transversal y no experimental sobre la prevalencia de Helicobacter pylori en pacientes pediátricos infectados por el virus de inmunodeficiencia humana (VIH), internados en el Hospicio San José, Santa Lucía Milpas Altas, Sacatepéquez, Guatemala, durante los meses de noviembre 2012 a enero 2013. La población completa fue conformada por 40 pacientes pediátricos de ambos sexos, entre los 3 y 12 años de edad, que padecen infección por VIH. Para el diagnóstico de infección por H. pylori se utilizó la detección de antígeno en heces por inmunocromatografía. Se encontró un resultado positivo en el 35\%, con una mayor incidencia en varones, correspondiendo al 71.4\% de los casos. En cuanto a la distribución por edades, el rango de edad con más casos positivos fue el comprendido entre los 7 y 8 años, con un $66.7 \%$ de los casos. La prevalencia de $H$. pylori en la población pediátrica VIH positiva estudiada fue mayor que la reportada en poblaciones similares, pero menor a la reportada en la población pediátrica VIH negativo. Se recomienda realizar un estudio multicéntrico para confirmar la prevalencia de $H$. pylori en la población pediátrica guatemalteca VIH positivo.

Palabras claves: niño, preescolar, estómago, morbilidad del niño

\begin{abstract}
quantitative, descriptive, transversal, non experimental study was conducted on the prevalence of Helicobacter A pylori in pediatric patients infected with the human immunodeficiency virus (HIV), who were admitted in the Hospice San Jose, Santa Lucia Milpas Altas, Sacatepequez, Guatemala, during November 2012 through January 2013. The study population included pediatric patients of both sexes, aged 3 to 12 years, who are infected with HIV. For the diagnosis of $H$. pylori infection an antigen stool test by immunochromatography was used. The population in the study period amounted to 40 patients in whom a positive result of 35\% was found, $71.4 \%$ from male patients. In the age distribution it was found that the age range with more positive cases $(66.7 \%)$ was between 7 and 8 years. The prevalence of $H$. pylori in the studied HIV positive pediatric population was higher than reported in similar populations, yet lower than reported in the HIV negative pediatric population. It is recommended that a multicenter study confirm the prevalence of H. pylori in the HIV positive Guatemalan pediatric population.
\end{abstract}

Keywords: kids, pre-school, stomach, infant morbidity 


\section{Introducción}

Helicobacter pylori es la principal especie de su género asociada a enfermedad en humanos, siendo capaz de colonizar de forma permanente el estómago de las personas. Es sumamente móvil debido a sus flagelos polares y gracias a poseer la enzima ureasa, puede sobrevivir a los ácidos gástricos (Dunn, Cohen, \& Blaser, 1997; Garza-Gonzalez, Perez-Perez, Maldonado-Garza, \& Bosques-Padilla, 2014). La transmisión de H. pylori puede ser por vía feco-oral u oral-oral, que en el caso de los niños puede adquirirse tanto de otros niños como de los padres (Rothenbacher et al., 1999). En los países en vías de desarrollo, la colonización o infección es adquirida a edades muy tempranas (Rowland et al., 2006) y aumenta significativamente en la adolescencia, reportándose en algunas poblaciones hasta un $91 \%$ de prevalencia (Daugule \& Rowland, 2008; Leandro et al., 2005; Robinson et al., 2002).

En el mundo se calcula la prevalencia de infección por H. pylori es mayor al 50\%, y en Guatemala, un estudio realizado en 184 menores de 3 años, encontró una prevalencia de inmunoglobulina $\mathrm{G}(\mathrm{IgG})$ contra $H$. pylori de 33\% (Oregel, 2002). Otro estudio realizado en 590 niños de San Juan Sacatepéquez, mostró una seroprevalencia de $20 \%$ en niños de 6 a 12 meses, la que se mantuvo estable incluso en los niños de 36 meses, con $25 \%$ (Steinberg et al., 2004). Se estima que en Guatemala el $65 \%$ de los adultos se encuentran infectados, mientras que la población entre 5 a 10 años tiene una prevalencia aproximada de 51\% (Hunt et al., 2010).

La infección por el virus de la inmunodeficiencia humana (VIH) es una epidemia que, según cálculos de la Organización Mundial de la Salud (OMS), afectó a 35 millones de personas alrededor del mundo en el 2013, siendo 1 de cada 10 casos en menores de 15 años; además, se estimaron 2.1 millones de casos nuevos, 240 mil de los cuales ocurrieron en menores de dicha edad 2013. Sin embargo, la mayor importancia radica en la mortalidad, pues unos 190 mil menores de 15 años fallecieron durante dicho período (Programa Conjunto de las Naciones Unidas sobre el VIH/sida, 2013). En Guatemala, en el año 2013 el Ministerio de Salud Pública y Asistencia Social (MSPAS) reportó 1,842 nuevos casos de infección por VIH; de estos, el 10\% ocurrió en la población pediátrica de 0 a 14 años (2014); por su parte, el Fondo de las Naciones Unidas para la Infancia (2013) en su reporte sobre Guatemala, estima que unos 2,757 niños de 0 a 14 años requieren tratamiento por esta infección.
La colonización por H. pylori en pacientes infectados por VIH parece ser igual o menor que en los niños sanos (Blecker et al., 1994; Lionetti et al., 1999), e incluso en los pacientes con estadios más avanzados de inmunocompromiso o aquellos que han recibido antibióticos recientemente (Hestvik et al., 2011). La menor colonización por $H$. pylori también se ha documentado por métodos invasivos, pues en 24 biopsias gástricas analizadas entre 1985 y 2002 en Santiago de Chile, la prevalencia fue de cero (Tassara, Alarcón, Larrañaga, Wu, \& Alvarez, 2003).

Por ser Guatemala un país con alta prevalencia de infección por $H$. pylori y al no contarse con estudios similares, se planteó como objetivo determinar la prevalencia de infección por $H$. pylori en niños con VIH del Hospicio San José, en los meses de noviembre 2012 a enero 2013.

\section{Materiales y métodos}

\section{Sujetos de estudio}

Se realizó un análisis cuantitativo, transversal, no experimental en pacientes pediátricos de ambos sexos y de 12 años o menos, con infección por VIH, que se encontraban internados en el Hospicio San José, Santa Lucía Milpas Altas, Sacatepéquez. Se analizó la población completa, conformada por 40 pacientes.

\section{Metodología}

Se practicó un consentimiento informado junto con las autoridades médico-administrativas del hospicio encargadas del cuidado de los pacientes, tras lo cual se proporcionó al personal de enfermería frascos estériles e instrucciones para la recolección de las muestras. Los datos generales y las muestras de heces de los pacientes fueron recolectados y proporcionados por el personal del hospicio. Las heces fueron analizadas por los investigadores utilizando el kit de detección de antígeno de H. pylori en heces por inmunocromatografía Pylori $\mathrm{K}$ Set ${ }^{\circledR}$ (Coris BioConcept), el cual reporta una sensibilidad de $96 \%$, especificidad de $98.2 \%$, valor predictivo positivo de $92.3 \%$ y un valor predictivo negativo de $99.1 \%$ al ser comparado con el diagnóstico por inmunoensayo enzimático convencional (Coris BioConcept, 2012). 


\section{Resultados}

Fueron obtenidos los datos de 40 participantes. La media de edad fue de 8.5 años, con una desviación estándar de 2.8. La región metropolitana fue el principal lugar de procedencia (Tabla 1)

Tabla 1

Características de la población estudiada, Hospicio San José, Guatemala

\begin{tabular}{lr}
\hline \multicolumn{1}{c}{ Característica } & $\mathrm{n}(\%)$ \\
\hline Edad & $12(30.0)$ \\
$3-6$ años & $28(70.0)$ \\
$7-12$ años & \\
Sexo & $18(45.0)$ \\
Femenino & $22(55.0)$ \\
Masculino & \\
Procedencia & $12(30.0)$ \\
Guatemala & $6(15.0)$ \\
Izabal & $5(12.5)$ \\
Escuintla & $4(10.0)$ \\
Suchitepéquez & $13(32.5)$ \\
Otros &
\end{tabular}

En la Tabla 2 puede observase que la prevalencia de $H$. pylori en la población fue de $35 \%$. El $71.4 \%$ de los casos positivos fue en el sexo masculino, con una razón de momios de 2.04 (intervalo de confianza 95\% de 0.5 a $7.6, p=0.29$ ).

Tabla 2

Casos positivos de H. pylori en la población estudiada, distribución por sexo, Hospicio San José, Guatemala

\begin{tabular}{lcc}
\hline Sexo & Positivos $(\mathrm{n})$ & Prevalencia $(\%)$ \\
\hline Masculino & 10 & 45.4 \\
Femenino & 4 & 22.2 \\
Total & 14 & 35.0 \\
\hline
\end{tabular}

La mayoría de los casos se detectó en los pacientes entre los 5 y 8 años de vida. Los grupos de 3 a 4 años y 9 a 10 presentaron las prevalencias más bajas. (Tabla 3).
Tabla 3

Casos positivos de H. pylori en la población estudiada, distribución por sexo, Hospicio San José, Guatemala

\begin{tabular}{cccc}
\hline $\begin{array}{c}\text { Edad } \\
\text { (años) }\end{array}$ & $\begin{array}{c}\text { Positivos } \\
(\mathrm{n})\end{array}$ & $\begin{array}{c}\text { Población } \\
(\mathrm{N})\end{array}$ & $\begin{array}{c}\text { Prevalencia } \\
(\%)\end{array}$ \\
\hline 3 a 4 & 1 & 5 & 20.0 \\
5 a 6 & 4 & 7 & 57.1 \\
7 a 8 & 4 & 6 & 66.7 \\
9 a 10 & 2 & 10 & 20.0 \\
10 a 11 & 3 & 12 & 25.0 \\
Total & 14 & 40 & 35.0 \\
\hline
\end{tabular}

\section{Discusión}

La prevalencia de $H$. pylori en el presente estudio fue de $35 \%$. En comparación con los resultados de Hetsvik y colaboradores (2011), quienes encontraron un $22.5 \%$ de casos positivos en niños hospitalizados de Uganda, en la población estudiada se encontró una prevalencia más alta, utilizando en ambos casos el mismo método de detección. También fue mayor que en el estudio realizado en Italia por Lionetti y colaboradores (1999), quienes reportaron una prevalencia de 17.7\% por detección de anticuerpos y de $20 \%$ con la prueba de urea; la discrepancia reportada fue explicada por la baja sensibilidad de las pruebas serológicas en pacientes con VIH, sobre todo en las etapas más avanzadas de la enfermedad. Por otra parte, Blecker y colaboradores (1994) en niños inmunodeficientes provenientes de Africa Central residentes en Bélgica y Tassara y colaboradores (2003) en niños inmunodeficientes en Santiago de Chile reportaron prevalencias de $0 \%$ en sus respectivos estudios, siendo el primero mediante la detección de IgG y el segundo por medio de biopsias gástricas; aunque las muestras en estos estudios fueron menores de 30 personas, ambos encontraron prevalencias bajas en comparación con la población seronegativa.

La prevalencia hallada fue menor a la de la población pediátrica en Guatemala, estimada en 51\% según Hunt y colaboradores (2010). La menor prevalencia encontrada en pacientes VIH positivo en comparación con la población general se puede explicar por factores propios del huésped relacionados con el VIH y la evolución natural de la enfermedad, incluidos hipoclorhidria e inadecuada respuesta inflamatoria de la mucosa, que pueden reducir la colonización exitosa por $H$. pylori (Edwards et al., 1991; Marano, Smith, \& Bonanno, 
1993). Oregel (2002) reportó una seroprevalencia de $33 \%$ en la población pediátrica, aunque su muestra consistió en pacientes menores de 3 años, grupo etario que no formó parte del presente estudio y el diagnóstico se basó en la prevalencia de anticuerpos séricos, por lo que se debe tomar en cuenta que la IgG a edades tempranas puede ser de origen transplacentario.

Si bien se encontró una mayor prevalencia en el sexo masculino, no se encontró significancia estadística. Leandro y colaboradores (2005) también encontraron una mayor prevalencia de casos positivos en el sexo masculino; la misma fue en la población pediátrica general y en España, un país con condiciones socioeconómicas distintas. Por el contrario, Hestvik y colaboradores (2011) reportaron una mayor prevalencia en pacientes pediátricos de sexo femenino VIH positivos de Ugamda, con una razón de momios no ajustada de 1.5 para el sexo femenino (intervalo de confianza $95 \%$ de 0.8 a $2.7, p=0.23$ ), diferencia que puede ser atribuida a la distinta relación masculino-femenino en las poblaciones estudiadas.

En cuanto a la distribución de casos por edad, la mayoría de resultados positivos para $H$. pylori se encontró entre los 5 y 8 años, mientras Hetsvik y colaboradores (2011) encontraron que el rango de edad de pacientes VIH positivos con mayor prevalencia fue el de 1 a 3 años de vida; se considera que la diferencia obedece a que en el presente estudio no se contó con menores de 3 años en la muestra, sin embargo es importante señalar que la colonización temprana también ocurre en pacientes seropositivos.

La distribución de casos por edades también fue distinta a la descrita en la literatura para la población pediátrica general, ya que posterior a los 6-7 años la prevalencia disminuyó, mientras que diversos autores describen que la misma permanece estable con el paso del tiempo o aumenta (Daugule \& Rowland, 2008; Leandro et al., 2005; Robinson et al., 2002; Rowland et al., 2006; Steinberg et al., 2004). Esta diferencia podría ser explicada por la necesidad de antibioticoterapia profiláctica o terapéutica en los pacientes seropositivos; también se ha sugerido que $H$. pylori necesita de un sistema inmune funcional para la colonización persistente de la mucosa gástrica, por lo que el continuo deterioro de la función inmune en esta población supondría una disminución progresiva de casos positivos (Nevin, Morgan, Graham, \& Genta, 2014).

Se concluyó que la prevalencia de $H$. pylori en la población pediátrica VIH positiva estudiada fue mayor que la reportada en poblaciones similares, pero menor a la reportada en la población pediátrica VIH negativo.
Se recomienda realizar un estudio multicéntrico para confirmar la prevalencia de H. pylori en la población pediátrica guatemalteca VIH positivo.

\section{Agradecimientos}

Los autores agradecen a todo el personal del Hospicio San José por su labor en la protección de los niños y por toda su colaboración para la obtención de datos del presente estudio. Se agradece a la Dra. Carmen de Tercero por su apoyo para la elaboración de este informe y por fomentar la investigación en los estudiantes de Medicina. Finalmente, se agradece a la Dirección de Investigación de la Facultad de Ciencias Médicas de la Universidad de San Carlos de Guatemala, por la revisión del presente artículo.

\section{Referencias}

Blecker, U., Keymolen, K., Lanciers, S., Bahwere, P., Souayah, H., Levi, J., \& Yvan, V. (1994). The prevalence of Helicobacter pylori positivity in human immunodeficiency virus-infected children. Journal of Pediatric Gastroenterology and Nutrition, 19(4), 417-420.

Coris BioConcept. (2012). Helicobacter pylori. Belgica: Autor. Recuperado de http://www.corisbio.com/ Products/Human-Field/Helicobacter-pylori.php

Daugule, I., \& Rowland, M. (2008). Helicobacter pylori infection in children. Helicobacter, 13(Supplement S1), 41-46. doi: 10.1111/j.15235378.2008.00632.x

Dunn, B. G., Cohen, H., \& Blaser, M. J. (1997). Helicobacter pylori. Clinical Microbiology Reviews, 10(4), 720-741.

Edwards, P. D., Carrick, J., Turner, J., Lee, A., Mitchell, H., \& Cooper, D. A. (1991). Helicobacter pyloriassociated gastritis is rare in AIDS: Antibiotic effect or a consequence of immunodeficiency? American Journal of Gastroenterology, 86(12), 1761-1764.

Fondo de las Naciones Unidas para la Infancia. (2013). UNICEF Annual report 2013 - Guatemala. Recuperado de http://www.unicef.org/about/ annualreport/files/Guatemala_COAR_2013.pdf

Garza-Gonzalez, E., Perez-Perez, G. I., Maldonado-Garza, H., \& Bosques-Padilla, F. (2014). A review of Helicobacter pylori diagnosis, treatment, and methods to detect 
eradication. World Journal of Gastroenterology, 20(6), 1438-1449. doi: 10.3748/wjg.v20.i6.1438.

Hestvik, E., Tylleskar, T., Ndeezi, G., Grahnquist, L., Olafsdottir, E., Tumwine, J. K., \& KadduMulindwa, D. H. (2011). Prevalence of Helicobacter pylori in HIV-infected, HAARTnaïve Ugandan children: A hospital-based survey. Journal of the International AIDS Society, 14, 34. doi:10.1186/1758-2652-14-34

Hunt, R. H., Xiao, S. D., Megraud, F., Leon-Barua, R., Bazzoli, F., van der Merwe, S., ... Krabshuis, J. H. (2010). Guías prácticas de la organización mundial de gastroenterología: Helicobacter pylori en los países en desarrollo. Gastroenterología Latinoamericana, 21(2), 165-181.

Leandro, S. V., Hernández, M., Torroba, L., Sánchez, F., Leandro, S. E., Gómez, A., Chueca, P. (2005). Infección por Helicobacter pylori en población infantil: Prevalencia, factores asociados e influencia sobre el crecimiento. Anales de Pediatría, 63(6), 489-494. doi: 10.1016/S16954033(05)70247-2

Lionetti, P., Amarri, S., Silenzi, F., Galli, L., Cellini, M., de Martino, M., \& Vierucci, A. (1999). Prevalence of Helicobacter pylori infection detected by serology and 13C-urea breath test in HIV-1 perinatally infected children. Journal of Pediatric Gastroenterology and Nutrition, 28(3), 301-306.

Marano, B. J., Smith, F., \& Bonanno, C. A. (1993). Helicobacter pylori prevalence in acquired immunodeficiency syndrome. American Journal of Gastroenterology, 88(5), 687-690.

Ministerio de Salud Pública y Asistencia Social. (2014). Informe nacional sobre los progresos realizados en la lucha contra el VIH y sida. Guatemala: Autor. Recuperado de http://www.unaids.org/ sites/default/files/country/documents//GTM_ narrative_report_2014.pdf

Nevin, D. T., Morgan, C. J., Graham, D. Y., \& Genta, R. M. (2014). Helicobacter pylori gastritis in HIVinfected patients: A review. Helicobacter, 19(5), 323-329. doi: 10.1111/hel.12131

Oregel, S. (2002). Prevalencia de anticuerpos séricos contra Helicobacter pylori en niños menores de 3 años de baja condición socioeconómica (Tesis de licenciatura). Universidad de San Carlos de Guatemala, Facultad de Ciencias Médicas, Guatemala.

Organización Mundial de la Salud. (2013). Global summary of AIDS epidemic. Ginebra, Suiza: Autor. Recuperado de http://www.who.int/hiv/ data/epi_core_dec2014.png?ua $=1$

Programa Conjunto de las Naciones Unidas sobre el $\mathrm{VIH} /$ sida. (2013). Informe mundial: ONUSIDA, informe sobre la epidemia mundial de sida 2013. Ginebra, Suiza: Autor. Recuperado de http:// www.unaids.org/sites/default/files/media_asset/ UNAIDS_Global_Report_2013_es_1.pdf

Robinson, L. G. E., Black, F. L., Lee, F. K., Sousa, A. O., Owens, M., Danielsson, D., ... Gold, B. D. (2002). Helicobacter pylori prevalence among indigenous peoples of South America. Journal of Infectious Diseases, 186(8), 11311137. doi: 10.1086/343803

Rothenbacher, D., Bode, G., Berg, G., Knayer, U., Gonser, T., Adler, G., \& Brenner, H. (1999). Helicobacter pylori among preschool children and their parents: evidence of parent-child transmission. Journal of Infectious Diseases, 179(2), 398-402. doi:10.1086/314595

Rowland, M., Daly, L., Vaughan, M., Higgins, A., Bourke, B., \& Drumm, B. (2006). AgeSpecific incidence of Helicobacter pylori. Gastroenterology, 130(1), 65-72. doi: http:// dx.doi.org/10.1053/j.gastro.2005.11.004

Steinberg, E. B., Mendoza, C. E., Glass, R., Arana, B., Lopez, M. B., Mejia, M., ... Luby, S. (2004). Prevalence of infection with waterborne pathogens: a seroepidemiologic study in children 6-36 months old in San Juan Sacatepequez, Guatemala. American Journal of Tropical Medicine and Hygiene, 70(1), 83-88.

Tassara, R., Alarcón, T., Larrañaga, C., Wu, E., \& Alvarez, A. M. (2003). Patología digestiva en niños infectados con el virus de inmunodeficiencia humana (VIH), en Santiago de Chile. Revista Médica de Chile, 131(1), 19-24. doi: 10.4067/ S0034-98872003000100003 